\title{
Radiation Induced Lung Injury: Prediction, Assessment and Management
}

\author{
Prashanth Giridhar, Supriya Mallick*, Goura Kishore Rath, Pramod Kumar \\ Julka
}

\begin{abstract}
Radiation induced lung injury has long been considered a treatment limiting factor for patients requiring thoracic radiation. This radiation induced lung injury happens early as well as late. Radiation induced lung injury can occur in two phases viz. early ( $<6$ months) when it is called radiation pneumonitis and late ( $>6$ months) when it is called radiation induced lung fibrosis. There are multiple factors that can be patient, disease or treatment related that predict the incidence and severity of radiation pneumonitis. Radiation induced damage to the type I pneumocytes is the triggering factor to initiate such reactions. Over the years, radiation therapy has witnessed a paradigm shift in radiation planning and delivery and successfully reduced the incidence of lung injury. Radiation pneumonitis is usually a diagnosis of exclusion. Steroids, ACE inhibitors and pentoxyphylline constitute the cornerstone of therapy. Radiation induced lung fibrosis is another challenging aspect. The pathophysiology of radiation fibrosis includes continuing inflammation and microvascular changes due to pro-angiogenic and profibrogenic stimuli resembling those in adult bronchiectasis. General supportive management, mobilization of airway secretions, anti-inflammatory therapy and management of acute exacerbations remains the treatment option. Radiation induced lung injury is an inevitable accompaniment of thoracic radiation.
\end{abstract}

Keywords: Lung cancer - pneumonitis - radiation - fibrosis

Asian Pac J Cancer Prev, 16 (7), 2613-2617

\section{Introduction}

Thoracic radiation can induce both acute and late effects on the lung. Radiation induced lung injury can occur in two phases viz. early ( $<6$ months) when it is called radiation pneumonitis and late ( $>6$ months) when it is called radiation induced lung fibrosis. In the earlier time crude treatment technique, lack of image guidance used to cause treatment limiting pulmonary toxicity. However, the radiation therapy has witnessed paradigm shift in radiation planning and delivery and successfully reduced the incidence of lung injury. Still, there is enough scope to improve upon and at the same time it is important to know the biology of lung injury as well as modality to combat such condition. There is limited literature reviewing the prevention, mitigation and treatment of acute and late radiation induced lung injury. This article is aimed to highlight the pathophysiology, predictive markers, imaging, prevention and treatment of radiation induced lung injury in the context of sophisticated radiation.

\section{Pathophysiology}

The lung is one of the most sensitive tissues to radiation. The most radiosensitive sub-unit of the lung is alveolarcapillary complex (Ghafoori et al., 2008). The alveolar epithelium consists of type 1 and type 2 pneumocytes with type 1 covering $90 \%$ of alveolar surface. After irradiation, it is thought that type 1 cells are denuded. Post radiation; the repair process of pneumocytes seems to be dysregulated (Zhao et al., 2009). The destruction of type 1 pneumocytes leads to increased secretion of growth factors and proteases and these, instead of repair cause degradation of extracellular matrix (Trott et al., 2004). Radiation also causes loss of barrier function by destruction of epithelial and endothelial cells. These changes also cause accumulation of inflammatory cells. In normal wound healing, this initial inflammatory response subsides but in radiation pneumonitis this inflammation induces a vicious cycle of further inflammation and fibrosis. The molecular pathogenesis of radiation induced lung injury has been briefly described below.

Radiation induces the release of cytokines within days to weeks. This first wave of cytokine release settles within two weeks. A second wave starts around 6-8 weeks post radiation (Rube et al., 2004; Fleckenstein et al., 2007). The second wave is associated with increases in oxidative damage to DNA and increase in ROS/RNS levels, hypoxia, decreased lung perfusion and increased TGF- B1 expression (Rube et al., 2004; Fleckenstein et al., 2007). The increased TGF B1 causes influx of fibroblasts and their conversion to myofibroblasts, thus causing lung fibrosis. This, in turn causes hypoxia which induces profibrogenic and proangiogenic factor release. 
This vicious cycle continues and leads to chronic lung disease (Vujaskovic et al., 2001).

\section{Predictive Markers in Radiation Pneumonitis:}

There are multiple factors that can be patient, disease or treatment related that predict the incidence and severity of radiation pneumonitis. A few meta-analyses have been done that identify such risk factors.

\section{Patient related}

Age $>65$ years (Palma et al., 2013), poor performance status, and presence of co-morbidities (Ivan et al., 2012) are associated with higher risk of pneumonitis.Poor pulmonary function tests (decreased FEV1 and poor DLCO) have been classically described to have increased risk of pneumonitis. But, in a recent study by Wang et al. (2013) poor pulmonary function test (PFT) at baseline did not correlate with increased risk of pneumonitis. This could be because of poorer oxygenation in patients with worse PFT and thus less radiation induced damage. Classically, smoking also has been described as a risk factor for radiation induced lung injury. In a literature based meta-analysis, ongoing smoking has been found to be a protective factor and a history of smoking showed a tendency towards protection from radiation pneumonitis (Ivan et al., 2012).

\section{Disease related}

Mid and lower lobe tumours of lung are associated with increased risk of symptomatic pneumonitis (Ivan et al.,2012). A number of factors may contribute to this. Mid and lower lobe tumours move more with respiration and thus may lead to more normal tissues getting irradiated. Also, ventilation and perfusion increases from apex to base in lungs. This can lead to increased oxygen free radical production with radiation and thus more damage. It has also been seen that, in supine position the gas exchange in lungs is reduced, more so in diseased lungs. This may lead to focal areas of hypoxia and increased production of angiogenic and fibrogenic factors. As most radiation treatments are in supine position, the difference in gas exchange can lead to increased lung injury. Radiation for breast cancer with tangential fields, carcinoma oesophagus and other mediastinal tumours are also associated with increased risk of pneumonitis.

\section{Treatment related}

In conventional radiation, a lung-PTV V20>30\% and lung-GTV V20 $>33 \%$ is predictive of symptomatic pneumonitis (Palma et al., 2013). A higher mean lung dose (>20 Gray) also predicts symptomatic pneumonitis (Tsujino et al., 2003) in conventional fractionation. Although fatal pneumonitis is uncommon, it is associated with large daily fractions (Palma et al., 2013). For stereotactic body radiotherapy, V20 $>10 \%$ and mean lung dose $>6$ Gy is associated with higher risk of grade 2-4 pneumonitis (Hideomi Yamashita et al., 2014). A number of drugs when administered are associated with increased risk of radiation induced lung injury. These include chemotherapeutic agents like bleomycin, cyclophosphamide, vincristine, taxanes, doxorubicin, dactinomycin, mitomycin, gemcitabine and targeted agents like erlotinib and bevacizumab (McDonald et al., 1995; Taghian et al., 2001; Rancati et al., 2003; Urbanic et al., 2009; Lind et al., 2012). Certain studies have shown that concurrent rather than sequential administrationof chemotherapy is associated with a higher risk of radiation pneumonitis (Taghian et al., 2001). But, in a recent literature based meta-analysis on radiation pneumonitis, sequential chemotherapy showed a trend towards higher risk of radiation pneumonitis than concurrent chemotherapy (Ivan et al., 2012). A number of factors could have contributed to this. Patients who receive sequential therapy are generally of a poor performance status and thus more prone to symptomatic radiation pneumonitis. The dose of chemotherapy is generally lower when used concurrently with radiation.It was also found that concurrent chemotherapy with paclitaxel-carboplatin was associated with a higher risk of pneumonitis than cisplatin-etoposide or other chemotherapy regimens (Palma et al., 2013).

\section{Molecular predictors}

The high incidence of radiation- induced damage, it's possible severity and limited treatment options creates a need to detect it in the sub-clinical phase. The identification of biological markers and genes for predicting pneumonitis will help us to tailor treatment accordingly. Serum TGF B1 levels tend to reach normal levels by the end of radiation in patients who do not develop pneumonitis. Patients with elevated post treatment serum TGF B1 above baseline exhibit a significantly higher risk of radiation induced lung injury (Anscher et al., 1998; Zhao et al., 2008). The measurement of plasma IL $1 \mathrm{a}$ and IL 6 levels can be applied as a predictive diagnostic tool for radiation pneumonitis.A persistently elevated IL 1a and IL 6 before, during and after radiation

Table 1. Radiological Grading Scale of Radiation Induced Pneumonitis (RP) (Kouloulias et al., 2013)

\begin{tabular}{ll}
\hline Grade & \multicolumn{1}{c}{ CT Findings } \\
\hline 0 & $\begin{array}{l}\text { No f indings. } \\
\text { Ground glass opacities without fuzziness of the subjacent } \\
\text { pulmonary vessels. }\end{array}$ \\
2 & $\begin{array}{l}\text { The findings may vary from ground glass opacities, } \\
\text { extending beyond the radiation field, to consolidation }\end{array}$ \\
3 & $\begin{array}{l}\text { Clear focal consolidation } \pm \text { elements of fibrosis. } \\
\text { Dense consolidation, cicatrisation atelectasis, (traction } \\
\text { bronchiectasis), significant pulmonary volume loss and } \\
\text { thickening. }\end{array}$ \\
\hline
\end{tabular}

Table 2. RTOG clinical grading scale of Radiation Pneumonitis (Cox et al., 1995)

\begin{tabular}{ll}
\hline Grade & \multicolumn{1}{c}{ CT Findings } \\
\hline 1 & $\begin{array}{l}\text { Mild dry cough not requiring medications } \\
\text { Cough requiring narcotic anti-tussives or dyspnoea not } \\
\text { at rest }\end{array}$ \\
3 & $\begin{array}{l}\text { Severe cough not controlled by medications. Dyspnoea } \\
\text { at rest. }\end{array}$ \\
4 & $\begin{array}{l}\text { Continuous oxygen or assisted ventilation } \\
5\end{array}$ \\
\hline
\end{tabular}


are predictive of radiation pneumonitis (Chen et al., 2005). Serum levels of ICAM 1 are persistently elevated in patients who develop radiation pneumonitis (Tsujisaki et al., 1991). In a study by Sasaki et al, serum SP-A and SP-D levels were elevated in patients who developed radiation pneumonitis. Serum SP-A and SP-D levels started to rise about three weeks into fractionated radiation therapy and reached a maximum at 5 to 6 weeks (50-60 Gy) in patients who developed radiation pneumonitis.Certain ATM gene polymorphisms have been associated with increased risk of radiation pneumonitis (Xiong et al., 2013).

\section{Imaging and Radiation Pneumonitis}

(a) Contrast enhanced computed tomography: CT can be used in the diagnosis of radiation pneumonitis. It may show ground glass opacities, consolidation, fibrosis, atelectactic cicatrisation, pulmonary volume loss or pleural thickening. (b) FDG-PET: Radiation causes increased FDG uptake in lungs. The increased uptake settles in 8-12 weeks after completion of radiotherapy (Juweid et al., 2007). Radiation pneumonitis results in persistently elevated FDG uptake. The linear distribution on both CT and PET scans distinguishes pneumonitis from infection or malignancy (Faria et al., 2007). In a study by Castillo et al, it was found that pre radiotherapy SUV95 of lung correlated with increased risk of radiation pneumonitis (Castillo et al., 2014). Thus, pre-treatment PET can be used to identify high risk patients and take preventive measures to reduce risk of severe or fatal pneumonitis; $(c)$ Newer imaging modalities like Hyperpolarised Helium-3 MR spectroscopy (Thind et al., 2012) and SPECT (Hoover et al., 2014) are under investigation for early diagnosis of

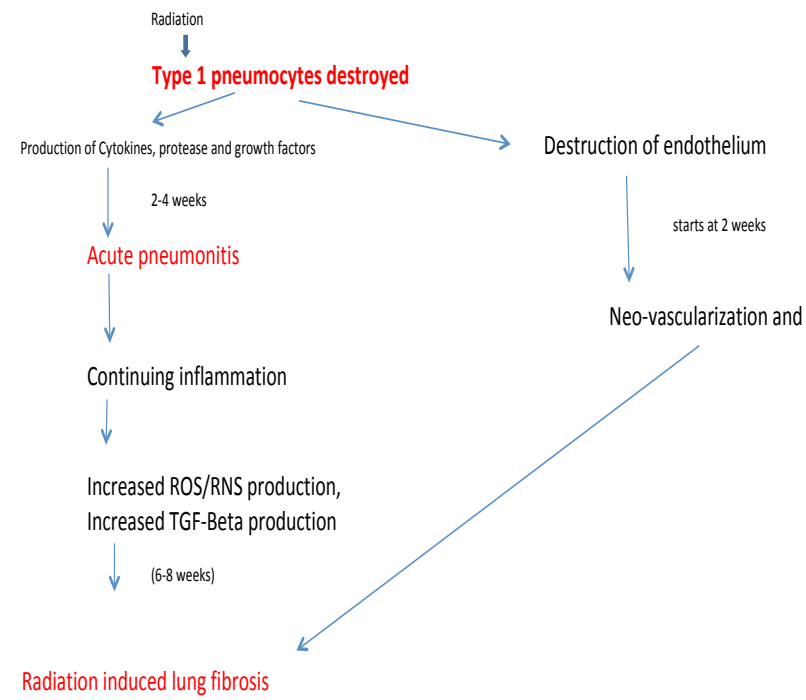

Figure 1. Pathophysiology

Radiation induced lung fibrosis

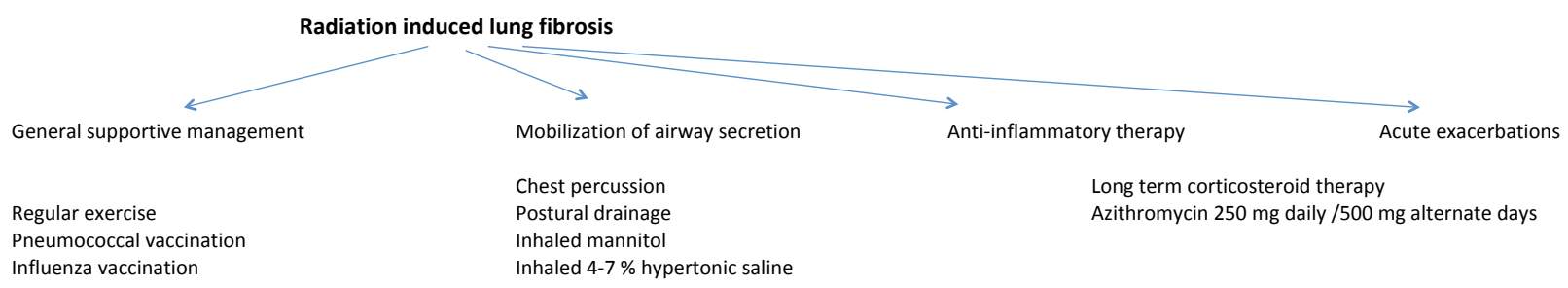

Figure 3. Radiation Induced Lung Fibrosis Relations
RP. SPECT detects perfusion deficits but lacks specificity in diagnosing radiation pneumonitis.

\section{Treatment of Radiation Pneumonitis}

i) Radiation pneumonitis is usually a diagnosis of exclusion. The differentials could be disease progression, concomitant infection, and exacerbation of chronic obstructive pulmonary disease, reactivation of latent tuberculosis or radiation pneumonitis.

ii) Presence of copious, purulent sputum, high grade fever and myalgia may be pointers toward an infective pathology. A complete blood count, throat swab, sputum culture/ sensitivity and chest $x$ ray may be warranted based on the clinical assessment.

iii) Steroids: Steroids form the mainstay of treatment of radiation pneumonitis. Once infection and disease progression are ruled out, the patient should receive oral prednisolone $1 \mathrm{mg} / \mathrm{kg}$ (max- $60 \mathrm{mg}$ ) for a period of two weeks followed by slow tapering over weeks (Bradley $\mathbf{J}$ et al., 2006). Abrupt stopping can lead to worsening of symptoms.

iv) ACE inhibitors: Multiple studies in rats have shown ACE inhibitors to be useful in mitigating radiation pneumonitis (Molteni A et al., 2000; Gao F et al., 2013). There is limited data regarding its efficacy in humans. Enalapril at low doses can be tried on an experimental basis. Patient's blood pressure should be monitored and bilateral renal artery stenosis should be ruled out before starting the drug. Enalapril may theoretically worsen cough due to bradykinin release and should be cautiously used. Enalapril is believed to decrease vascular remodelling and decrease levels of TGF B and thus decrease lung fibrosis (Medhora et al., 2012).

v) Pentoxyphylline: Multiple studies in rats have shown that pentoxyphylline reduces rate of alveolar septal thickening and lung fibrosis (Osterreicher et al., 2001). It can be started at a dose of $400 \mathrm{mg}$ thrice daily and continued for a period of two weeks. No robust data

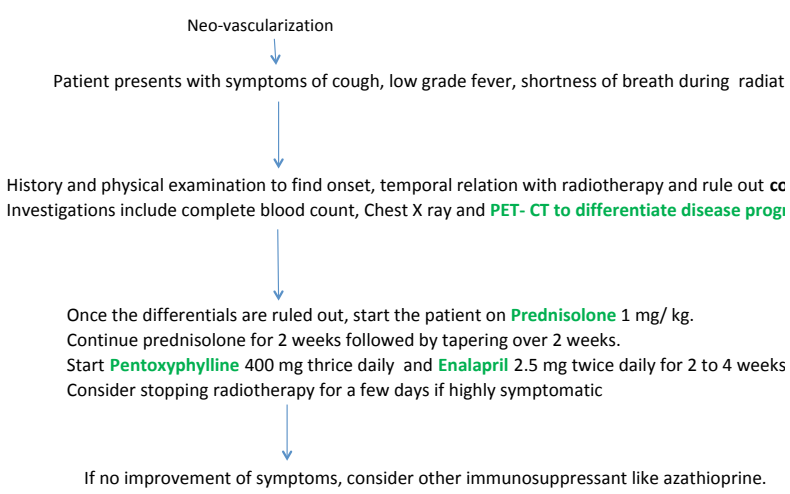

Figure 2. Acute Pneumonitis Management 
is available to support its use but it can be tried on an experimental basis.

vi) Anti-oxidants: Vitamin A, C and E supplements can be added as supportive therapy. vii) Supplemental Oxygen therapy: oxygen therapy can be used to reduce the increased work of breathing. viii) Other immunosuppressant like azathioprine can be tried in refractory cases.

\section{Management of Radiation Induced Lung Fibrosis}

There are no defined protocols or guidelines for the management of radiation induced lung fibrosis. The pathophysiology of radiation fibrosis includes continuing inflammation and microvascular changes due to proangiogenic and pro-fibrogenic stimuli resembling that of adult bronchiectasis. The problems include decreased muco-ciliary clearance, plugging of airways, chronic infection with acute exacerbations and worsening fibrosis. The symptoms include a dry non-productive cough, chest pain, dyspnea, decreased effort tolerance and fatigue. The management includes:

1. General supportive management

2. Mobilization of airway secretions

3. Anti-inflammatory therapy

4. Management of acute exacerbations

General supportive management includes good nutrition, regular exercise and vaccinations. Pneumococci and influenza viruses have been shown to cause life threatening infections in patients with underlying lung diseases like bronchiectasis. Pneumococcal and influenza vaccines should be considered in patients with lung fibrosis.

Mobilization of airway secretion is a crucial step in the management as excessive mucus can lead to mucus plug formation, increased bacterial load and recurrent infections. However, cough is less effective in clearing secretions as the mucus produced is dehydrated and highly viscus due to radiation induced damage. Airway secretion mobilization can be tried with chest percussion, postural drainage, controlled cough, inhaled mannitol and hypertonic saline. Studies have shown that inhalation of dry powder mannitol leads to increased hydration of mucus secretion and better clearance. The mechanism of action is not clear. Hypertonic saline at $4-7 \%$ also produces similar effects.

Anti-inflammatory therapy includes corticosteroids and macrolide antibiotics. Corticosteroids are the mainstay of management in acute radiation pneumonitis but their role in established lung fibrosis is not clear. Caution should be exercised while using them on a long term basis in view of a number of side effects. Macrolides are thought to accumulate in inflammatory cells and reduce the production of damage producing oxygen radicals. A number of studies have shown that macrolides reduce the number of exacerbations in bronchiectasis (Albert RK et al., 2011). No study specific to radiation induced fibrosis is available. Azithromycin at a dose of $250 \mathrm{mg}$ daily or 500 $\mathrm{mg}$ on alternate days can be considered for long term use.
The choice of antibiotic for acute exacerbations is not clear. Pseudomomas aeruginosa has been shown to cause acute exacerbations in bronchiectasis. Antibiotic combinations used should cover gram negative, gram positive and anaerobic organisms. The overall prognosis of established lung fibrosis is poor and patients progressively develop right heart failure.

\section{Conclusions}

Radiation induced lung injury is an inevitable accompaniment of thoracic radiation. Better radiation planning, incorporation of newer technology, use of predictive markers and overall clinical judgement is necessary to decrease the incidence and severity of radiation induced lung injury. Further research into the molecular pathology will help identify targets to halt and / or revert lung fibrosis. There is limited literature comprehensively reviewing radiation induced lung injury. This article is aimed to give an insight into the prediction and management of radiation induced lung injury.

\section{References}

Albert RK, Connett J, Bailey WC, et al (2011). Azithromycin for prevention of exacerbations of COPD. N Engl J Med, 365, 689-98.

Anscher MS, Kong FM, Andrews K, et al (1998). Plasma TGF $\mathrm{B} 1$ as a predictor of radiation pneumonitis. Int $\mathrm{J}$ Radiat Oncol Biol Phys, 41, 1029-35.

Bradley J, Movsas B (2006). Radiation pneumonitis and esophagitis in thoracic irradiation. In: Small W, Woloschak GE, eds. Radiation Toxicity: a Practical Guide. New York, NY: Springer Science+Media Business, Inc, 43-52.

Castillo R, Pham N, Ansari S, et al (2014). Pre-radiotherapy FDG PET predicts radiation pneumonitis in lung cancer. Radiat Oncol, 9, 74.

Chen Y, Ollivier H, Williams J, et al (2005). IL 1a and IL 6 application to the predictive diagnosis of radiation pneumonitis. Int J Radiat Oncol Biol Phys, 62, 260-6.

Cox JD, Stetz J, Pajak TF (1995). Toxicity criteria of the radiation therapy oncology group (RTOG) and the European organization for research and treatment of cancer (EORTC). Int J Radiat Oncol Biol Phys, 31, 1341-6.

Faria S, Lisbona R, Stem J, et al (2007). Is post-treatment FDG$\mathrm{PET} / \mathrm{CT}$ useful in differentiating tumor from fibrosis after curative radiation therapy (RT) alone for lung cancer? Int $J$ Radiat Oncol Biol Phys, 69, 519-20.

Fleckenstein K, Zgonjanin L, Chen L (2007). Temporal onset of hypoxia and oxidative stress after pulmonary irradiation. Int J Radiat Oncol Biol Phys, 68, 196-204.

Gao F, Fish BL, Moulder JE, et al (2013). Enalapril mitigates radiation-induced pneumonitis and pulmonary fibrosis if started 35 days after whole-thorax irradiation. Radiat Res, 180, 546-52.

Ghafoori P, Marks LB, Vujaskovic Z, Kelsey CR (2008). Radiation-induced lung injury. Assessment Manage Prev Oncol, 22, 37-47.

1Hideomi Yamashita, Wataru Takahashi; Akihiro Haga; Keichi Nakagawa (2014). Radiation pneumonitis after SBRT for lung cancers. W J radiology, 6, 708-15.

Hoover DA, Reid RH, Wong E, et al (2014). SPECT-based functional lung imaging for the prediction of radiation pneumonitis: a clinical and dosimetric correlation. $J$ Med Imaging Radiat Oncol, 58, 214-22. 
Ivan R Vogelius, Soren M Bentzen (2012). A literature based Meta analyses of clinical risk factors for development of radiation induced pneumonitis. Acta Oncol, 51, 975-83.

Juweid ME, Stroobants S, Hoekstra OS, et al (2007). Use of positron emission tomography for response as $\neg$ sessment of lymphoma: consensus of the imaging subcommittee of international harmonization project in lymphoma. $J$ Clin Oncol, 25, 571-8.

Kouloulias V, Zygogianni A, Efstathopoulos E, et al (2013). Suggestion for a new grading scale for radiation pneumonitis based on radiological findings of computerized tomography: Correlation with clinical and radiotherapeutic parameters in lung cancer patients. Asian Pac J Cancer Prev, 14, 2717-22.

Lind JS, Senan S, Smit EF (2012). Pulmonary toxicity after bevacizumab and concurrent thoracic radiotherapy observed in a phase I study for inoperable stage III non-small-cell lung cancer. J Clin Oncol, 30, 104-8.

McDonald S, Rubin P, Phillips TL, et al (1995). Injury to the lung from cancer therapy: clinical syndromes, measurable endpoints, and potential scoring systems. Int J Radiat Oncol Biol Phys, 31, 1187.

Medhora M, Gao F, Jacobs ER, Moulder JE (2012). Radiation damage to the lung: mitigation by angiotensin-converting enzyme (ACE) inhibitors. Respirology, 17, 66-71.

Molteni A, Moulder JE, Cohen EF, et al (2000). Control of radiation-induced pneumopathy and lung fibrosis by angiotensin-converting enzyme inhibitors and an angiotensin II type 1 receptor blocker. Int J Radiat Biol, 76, 523-32.

Osterreicher J, Mokry J, Navrátil L, et al (2001). The alveolar septal thickness and type II pneumocytes number in irradiated lungs, time expression and the effect of pentoxifylline. Acta Medica, 44, 15-9.

Palma DA, Senan S, Tsujino K, et al (2013). Predicting radiation pneumonitis after chemoradiotherapy of lung cancer: an international individual patient data meta-analysis. Int $J$ Radiat Oncol Biol Phys, 85, 444-50.

Rancati T, Ceresoli GL, Gagliardi G, et al (2003). Factors predicting radiation pneumonitis in lung cancer patients: a retrospective study. Radiother Oncol, 67, 275.

Rube CE, Wilfert F, Palm J, et al (2004). Irradiation induces a biphasic expression of pro-inflammatory cytokines in the lung. StrahlentherOnkol, 180, 442-8.

Taghian AG, Assaad SI, Niemierko A, et al (2001). Risk of pneumonitis in breast cancer patients treated with radiation therapy and combination chemotherapy with paclitaxel. $J$ Natl Cancer Inst, 93, 1806.

Thind K, Chen A, Friesen-Waldner L, et al (2012). Detection of radiation-induced lung injury using hyperpolarized (13) $\mathrm{C}$ magnetic resonance spectroscopy and imaging. Magn Reson Med, 70, 601-9.

Trott KR, Herrmann T, Kasper M, et al (2004). Target cells in radiation pneumopathy. Int J Radiat Oncol Biol Phys, 58, 463-469.

Tsujino K, Hirota S, Endo M, et al (2003). Predictive value of dose-volume histogram parameters for predicting radiation pneumonitis after concurrent chemoradiation for lung cancer. Int J Radiat Oncol Biol Phys, 55, 110-5.

Tsujisaki M, Imai K, Irata H, et al (1991). Detection of circulating ICAM1 in malignant diseases. Clin Exp Immunology, 81, 3-8.

Urbanic JJ, Lally B, Blackstock AW (2009). The best-laid plans ...often go awry...". J Thorac Oncol, 4, 783.

Vujaskovic Z, Anscher MS, Feng QF. et al (2001). Radiationinduced hypoxia may peRILIetuate late normal tissue injury. Int J RadiatOncolBiolPhys, 50, 851-5.

Wang J, Cao J, Yuan S, Ji W (2013). Poor baseline pulmonary function may not increase the risk of radiation induced lung toxicity. Int J Radiat Oncol Biol Phys, 85, 798-804.

Xiong H, Liao Z, Liu Z, et al (2013). ATM polymorphisms predict severe radiation pneumonitis in ptients with nonsmall cell carcinoma lung treated with definitive radiation therapy. Int J Radiat Oncol Biol Phys, 85, 1066-73.

Zhao W, Robbins ME (2009). Inflammation and chronic oxidative stress in radiation-induced late normal tissue injury: Therapeutic implications. Curr Med Chem, 16, 130-43.

Zhao L, Sheldon K, Chen M, Yin MS (2008) predictive role of plasma TGF B1 during radiation therapy for radiation lung toxicity deserves further study in patients with NSCLC. Lung Cancer, 59, 232-9. 